RAE-IC, Revista de la Asociación Española de Investigación de la Comunicación vol. 8, núm. 16 (2021), 142-167 ISSN 2341-2690

Recibido el 3 de septiembre de 2021 DOI: https://doi.org/10.24137/raeic.8.16.8 Aceptado el 20 de septiembre de 2021

\title{
La representación LGTBIQ+ en la serie infantil Steven Universe
}

The LGTBIQ+ representation in the children's show Steven Universe

Godoy, Marina

Universitat Autònoma de Barcelona (UAB)

marina.godoy.contente@gmail.com

García-Muñoz, Núria

Universitat Autònoma de Barcelona (UAB)

nuria.garcia@uab.cat

Forma de citar este artículo:

Godoy, M. y García-Muñoz, N. (2021). La representación LGTBIQ+ en la serie infantil Steven Universe. RAE-IC, Revista de la Asociación Española de Investigación de la Comunicación, 8(16), 142-167. https://doi.org/10.24137/raeic.8.16.8

\section{Resumen:}

Este artículo presenta los principales resultados de un estudio sobre la representación de personajes del colectivo LGTBIQ+ en la serie de animación infantil Steven Universe, creada por Rebecca Sugar y distribuida por Cartoon Network Studio. La ficción ha sido y sigue siendo el género de entretenimiento más valioso para los mercados y también para las audiencias. Por ello el estudio de los personajes ficcionales es esencial para conocer los roles representados sobre comunidades concretas. A través de un estudio 
de los personajes de la serie analizaremos si las ficciones seriadas infantiles actuales refuerzan el discurso cisheteropatriarcal o si, por el contrario, existen alternativas mediante las cuales se representan otras realidades, con una mirada más inclusiva en la que las perspectivas de género y LGTBIQ+ se integren en la narración.

Palabras clave: LGTBIQ+, serie infantil, animación, estereotipos, género.

\section{Abstract:}

This article unveils the main results of a study on the representation of characters of the LGTBIQ+ collective in the children's animated series Steven Universe, created by Rebecca Sugar and distributed by Cartoon Network Studio. Fiction has been and continues to be the most valuable entertainment genre for markets and also for audiences. For this reason, the study of fictional characters is essential to know the roles played in specific communities. Through a study of the characters in the series, we will analyze whether current children's serial fictions reinforce the cisheteropatriarchal discourse or if, on the contrary, there are alternatives through which other realities are represented, with a more inclusive view in which the perspectives of gender and LGTBIQ+ are integrated into the narrative.

Keywords: LGTBIQ+, children's serial fiction, animation, stereotype, gender.

\section{INTRODUCCIÓN}

La representación de personajes del colectivo $\mathrm{LGTBIQ}+{ }^{1}$ en la ficción seriada depende de diversos factores $y$, algunos de ellos, son el protagonismo que tengan dichos personajes en un producto audiovisual, el propio género audiovisual y evidentemente el público objetivo al que se destine cada ficción.

La investigación que presentamos trata sobre la representación del colectivo LGTBIQ+ en la ficción seriada infantil a través del estudio de caso de la serie de animación Steven

\footnotetext{
${ }^{1}$ LGTBIQ+: "Siglas de los términos lesbiana, gay, trans, bisexual, intersexual y queer, a las que se añade el signo + para aludir al resto de diversidades sexuales y de género." (Castro y Domingo, 2019).
} 
Universe. Dicha serie fue creada por Rebecca Sugar y distribuida por Cartoon Network Studio, perteneciente al grupo Warner Bros. Television Distribution y dirigida a un público de aproximadamente entre 4 y 11 años. Fue emitida por primera vez en mayo del 2013 y finalizó en enero del 2019.

Se trata de una serie de extraordinaria innovación respecto a la temática LGTBIQ+, ya que cuenta con personajes principales y secundarios con identidades de género y sexuales poco frecuentes en el ámbito audiovisual y para este target en concreto (Ziegler y Richards, 2020; Dunn, 2016; Aguado-Peláez, 2019; Robles, 2020). La aproximación a la serie infantil intenta conocer si esta ficción norteamericana refuerza el discurso cisheteropatriarcal $^{2}$ o si, por el contrario, existen alternativas mediante las cuales se representan otras realidades, con una mirada más inclusiva en la que las perspectivas de género y LGTBIQ+ se integren en la narración.

Este estudio se enmarca en el análisis llevado a cabo en el trabajo final de Máster Oficial en Contenidos de Comunicación Audiovisual y Publicidad de la Universitat Autònoma de Barcelona.

\section{MARCO TEÓRICO}

El valor de los estereotipos mostrados en la ficción se ha redimensionado a partir de la oferta multicanal, el consumo OTT y la participación activa de las comunidades en las redes sociales. El papel de la ficción en la transmisión de estereotipos representados es una realidad validada por numerosas investigaciones (Gerbner et al., 2002; Peterson y Peters, 1983; Signorielli y Bacue, 1999; Lacalle y Gómez, 2016; García-Muñoz y Martínez, 2009). El actual ecosistema relativo a la industria audiovisual y a las formas de consumo de ficción ha provocado una multiplicación de contenidos y contextos de la recepción que inciden, directa e indirectamente, en los estereotipos que generamos en materia de género y LGTBIQ+.

\subsection{LA REPRESENTACIÓN LGTBIQ+ EN LA FICCIÓN TELEVISIVA}

\footnotetext{
2 Cisheteropatriarcado: "Ingrediente por el cual los hombres, la heterosexualidad y las personas cis son consideradas la norma y tienen mayor reconocimiento, prestigio y valoración social y por ello supremacía sobre otros géneros y sobre otras orientaciones sexuales" (Sánchez, 2019, p. 35).
} 
El uso de estereotipos en ficción podría representar un arma de doble filo: si bien por una parte es innegable que facilita la rápida comprensión del espectador (Alfeo, 2011), también corre el riesgo de "convertirse en recurso fácil para ridiculizar o denigrar" (Cuesta y Menéndez, 2008, p. 68).

Como es obvio, esto representa un desafío para los autores, dado que las construcciones de género son muy complejas y fácilmente pueden herir la sensibilidad de algún sector del público.

Se puede crear un personaje de género masculino (identificado con el género masculino) según los estereotipos que la sociedad asocia con el género femenino (y cumplirlos) y viceversa (un personaje femenino puede responder a estereotipos masculinos). Un ejemplo claro es el caso de Cameron de la serie Modern Family: un personaje masculino que responde a estereotipos femeninos (Martín-Sánchez, Hernández-Herrarte y Fernández-Ramos, 2020). Cuando se aplican estas características consideradas "femeninas" a personajes masculinos (sobre todo en el género de la comedia), el personaje en cuestión suele formar parte del colectivo LGTBIQ+.

Otra herramienta teórica para analizar personajes LGTBIQ+ es la propuesta por Alfeo (2011), que añadió a su estudio la categoría de "representación hacia el rasgo LGBT en diferentes ámbitos" (Alfeo, 2011, p. 76) así como también un análisis de carácter más espacio-temporal y centrado en la acción diferencial (Alfeo, 2011, p. 78).

Alfeo insiste en la necesidad de dar a conocer las realidades LGTB y en la importancia que juega la ficción para conseguirlo. Explica que muchas veces ese es el único contacto que tienen los espectadores con esta realidad (Alfeo, 2011).

Los medios erigen directamente modelos de superioridad social, con lo que perjudican de manera directa a sectores minoritarios como el colectivo LGTBIQ+ (Collins, 2009). Heredia (2016) afirma que ahora son nuevas minorías, ya sean raciales, el colectivo 
LGTBIQ+ u otras, las que ocuparán este sitio que hasta ahora ocupaban las mujeres cisheteronormativas ${ }^{3}$ dentro de la ficción.

Los contenidos audiovisuales de consumo masivo cuyo elenco protagónico está formado (al menos parcialmente) por personas con identidades de género y orientaciones sexuales disidentes no ha dejado de crecer en los últimos años. Encontramos múltiples ejemplos en series como Orange is the New Black, Sense8, Pose, La Veneno, Dancing with the Stars o Transparent.

Pero este fenómeno no es tan nuevo como se podría presuponer, ya que también podemos indicar personajes LGTBIQ+ en series como Desperate Housewives, Buffy the Vampire Slayer o Six Feet Under, por ejemplo (Chambers, 2009). Aunque es un hecho que en la última década se ha acentuado particularmente la presencia de estos (GLAAD, 2020-2021).

Se detecta igualmente una tendencia creciente a la representación más inclusiva. Esto se ve claramente en Orange is the New Black (Netflix, 2013-2019), que narra la vida cotidiana de varios de los colectivos marginados de la sociedad en uno de los entornos más hostiles, un centro penitenciario (Martínez y Aguado-Peláez, 2017). Fue una de las primeras series en acercar al público la realidad de distintas minorías étnicas, sexuales, de género y sociales en un contexto de particular violencia con las jerarquías sociales y de poder que comporta.

En mayor o menor medida esta tendencia creciente a una representación televisiva más inclusiva está presente igualmente en series como Game of Thrones, Orphan Black o Sense8 (Martínez y Aguado-Peláez, 2017). Esta última serie resulta clave a la hora de entender el desarrollo y la evolución de los estereotipos ligados al género y la sexualidad de los personajes de ficción en la actualidad, puesto que rompe con los estereotipos tradicionales de los personajes LGTBIQ+ y pone en pantalla "una amplia variedad de

\footnotetext{
${ }^{3}$ Cisheteronormatividad: "Hace referencia a un sistema de representaciones que asigna significado (identidad, valor, prestigio, ubicación en la jerarquía social, etc.) a los individuos en una sociedad determinada (De Lauretis, 1989). En este contexto, ser representado y representarse como varón o mujer implica asumir la totalidad de los efectos de esos significados. No obstante, dentro de ese sistema de representaciones también se construyen otras identidades de género por fuera del binomio "varón" y "mujer" que "ocuparán posiciones diferenciales de acuerdo con las jerarquizaciones y a las estrategias de poder de cada momento histórico determinado"” (Zani, 2019, p. 340).
} 
identidades sexuales $y$, lo que es mas importante, una total normalización de la visibilidad de las relaciones sexuales entre dos hombres o dos mujeres, y hombre y mujer." (Fernández, 2017, p. 70). Más aún, series como esta también contribuyen a la construcción de discursos sociales sobre sexo, género y sexualidad, y conducen a que la sociedad los codifique y normalice de la misma manera que la heteronormatividad y la cisnormatividad (Fellner, 2017, p. 3).

Algunas productoras de contenidos seriados infantiles, como Cartoon Network, llevan años intentando incluir este tipo de personajes en sus contenidos audiovisuales. Aún así, cabe destacar que si bien es cierto que en la serie animada Powerpuff Girls la figura de Him se correspondía con varios parámetros de la estética queer ${ }^{4}$, no dejaba de ser un personaje fuertemente estereotipado con tacones altos, muy maquillado, vestido de forma frívola, entre otros aspectos. Por otra parte, no dejaba de encarnar al villano de la serie y eso es algo que el lector siempre debe tener en cuenta, dadas las connotaciones negativas que suele tener ese papel (Santos, Peres y Maranho, 2019).

La primera temporada de la animación The Amazing World of Gumball (Cartoon Network, 2011-2019) marcó otro hito en el camino hacia la normalización de personajes LGTBIQ+ en los contenidos para niños y niñas al incluir una variedad de figuras LGTBIQ+ que ilustran ampliamente la diversidad a través de personajes de todo tipo, desde un gato hasta una banana, pasando por una nube e incluso un pez (Chiu y Chang, 2018). Esta representación implica igualmente que muchos de estos personajes no tengan un género fácilmente identificable, lo que complica el estudio de los mismos siguiendo los parámetros utilizados en este trabajo.

\subsection{STEVEN UNIVERSE Y LOS ESTEREOTIPOS INVESTIGADOS}

La presencia de identidades sexuales y de género disidentes llegó también a contenidos para niños y niñas. De hecho, esta temática lleva años siendo explorada a través de

\footnotetext{
${ }^{4}$ Queer: "La teoría queer es heredera del pensamiento de Foucault, el posfeminismo anticolonial y del movimiento gay y lesbiano que pone en duda la estabilidad de las identidades en el sistema sexo/género. Asimismo, se oponen a aquella otra parte que tiende al integracionismo y genera formas de opresión desplazando a grupos minorizados. Lo 'queer' imagina subjetividades abiertas frente al modelo ontológico dual y rechaza los efectos normalizadores de cualquier formación identitaria (Preciado en Universidad Internacional de Andalucía (UNIA), 2003)." (Domínguez-Benítez, 2020, p. 92).
} 
productos audiovisuales infantiles como Sailor Moon o Ouran High School Host Club en países orientales (Delamorclaz, 2018). Mientras, en occidente los personajes LGTBIQ+ "humanos" permanecían prácticamente ausentes hasta la aparición de Steven Universe (2013), en la que se introduce una innovación relevante al incluir personajes de género no binario ${ }^{5}$ y representaciones que indican la constante transformación del cuerpo (Delamorclaz, 2017, p. 2).

Si bien es cierto que aquí nos focalizaremos en la presencia de contenidos LGTBIQ+ en Steven Universe, no hay que perder de vista que, como bien explicó Crenshaw: "All oppressions are related between them, there is no way to solve them separately." (1991).

Una de las mayores riquezas de este contenido es la variedad respecto a las problemáticas sociales que aborda. Para ello Aguado-Pelaéz (2019) plantea el concepto de matriz de dominación aplicado a Homeworld, el planeta del cual la mayoría de personajes mágicos (personajes-gemas ${ }^{6}$ ) provienen. Así, Homeworld se manifiesta en esta serie como una figura espacial opuesta al planeta Tierra (concretamente Beach City, que es donde viven Steven y sus compañeros y donde sucede la acción en la Tierra). En otras palabras, se plantea como un universo totalmente distópico, un régimen totalitario dirigido por un matriarcado basado en la supremacía de algunos personajesgemas y en un sistema de clases o de castas. (Aguado-Peláez, 2019, p. 213).

La relevancia de Steven Universe reside igualmente en el hecho de abordar temas de género que no habían sido tratados anteriormente en contenidos infantiles, acentuando la inclusión de enfoques alternativos sobre el género y la orientación sexual de los personajes (Robles, 2020). Los personajes-gemas no tienen sexo ni género, y aunque su apariencia es feminizada, no se las puede concebir como estrictamente binarias (Robles, 2020, p. 75). Más aún, sus rasgos (sus actitudes, los estereotipos a los que responden)

\footnotetext{
${ }^{5}$ No-binario o género fluido: “Un término de identidad para describir a una persona cuya identidad no puede describirse con los términos binarios asociados con varón/hombre o mujer/hembra; una identidad de género que se ubica en alguna parte del espectro del género." (CHAT, 2021).

${ }^{6}$ En Steven Universe los personajes-gemas son aquellos provenientes de Homeworld. Cada personaje-gema es una piedra preciosa que exterioriza su luz para formar un cuerpo físico. Se caracterizan por poseer un inmenso conocimiento en la tecnología y fuerza sobrehumana (mágica) que emplean para poder conquistar otros mundos en pos de su reino.
} 
no suelen corresponderse con los que se usan normalmente para estereotipar a un personaje de género femenino en una animación infantil (Robles, 2020).

La construcción de escenarios y situaciones concretas son el componente clave y principal para la inclusión de la realidad LGTBIQ+ en la serie (Dunn, 2016; Cooley, 2020).

Las "fusiones" permiten a las gemas, por ejemplo, unirse mágicamente entre dos o más para pasar a formar un solo ser, más fuerte, que reúna los poderes de todas ellas, conformando un proceso clave. Dunn (2016) habla de ellas como "the most useful for queer representation in Steven Universe". La fusión de gemas sintetiza así el potencial de la inclusión y solidaridad. Es igualmente un aspecto clave para comprender la obra en clave LGTBIQ+.

Nacieron inicialmente con una finalidad bélica pero que a lo largo de los episodios fueron evolucionando y adoptando nuevas connotaciones y con la posibilidad de producirse simbiosis por razones sentimentales o por simple solidaridad.

Los elementos del universo queer están presentes en prácticamente toda la serie, tanto en la caracterización de los personajes como en la ideología que se promueve gracias a este programa. En ese sentido, varios autores como Pitre (2020), Alfeo (2011) o Martí (2011) coinciden reiteradamente en la necesidad de contenidos tanto para adultos como infantiles que traten la temática queer como un medio para familiarizar a la sociedad con este colectivo y normalizar su presencia.

Puede considerarse que la serie Steven Universe responde a esta demanda ya que, como apuntan García-García y Cornelio-Marí: "aunque las gemas no son humanas, sino proyecciones físicas creadas por cristales mágicos, se muestran siempre como personajes humanoides con forma femenina. Por ello, toda relación romántica entre gemas es eminentemente homosexual en su representación visual." (García-García y Cornelio-Marí, 2019, p. 4).

Las aportaciones sobre la serie de animación Steven Universe revelan la importancia de esta ficción televisiva dirigida al público infantil y juvenil en relación con la representación de sus personajes. Por ello, la investigación presentada en este trabajo 
tiene como objeto ahondar en los estereotipos LGTBIQ+ retratados a través de los personajes principales y parte de los secundarios.

Las contribuciones de los antecedentes sobre el tema investigado nos han permitido centrar los objetivos del estudio en cuatro preguntas de investigación. La primera, ¿qué características presentan los personajes heterosexuales y LGTBIQ+?, la segunda ¿qué diferencias y similitudes tiene la representación de personajes cisheterosexuales y personajes del colectivo LGTBIQ+? , la tercera ¿cómo son los contextos representados?; y, finalmente, ¿̇uáles son los roles dominantes en el retrato de los personajes?.

\section{METODOLOGÍA}

La estrategia metodológica se ha desarrollado a partir de la técnica de análisis de contenido de la muestra seleccionada y conformada por los personajes principales y secundarios de la primera temporada de la serie de animación Steven Universe. Los parámetros en relación con la temporada emitida responden a tres criterios principales. En la primera temporada se asientan conceptos básicos de la serie y se introducen los personajes, tanto principales como secundarios. Además, en el caso concreto de Steven Universe la primera temporada es la más extensa de las cinco que conforman la serie, ya que cuenta con 52 episodios a diferencia de los 20-30 registrados en otras temporadas. Cabe indicar también que la primera temporada seleccionada fue la que consiguió un mayor éxito, tanto por parte de las audiencias como por parte de la crítica. El análisis realizado corresponde, por tanto, a los 52 episodios y a los roles representados en relación con los personajes indicados en la siguiente tabla: 
Tabla 1. Personajes de la muestra

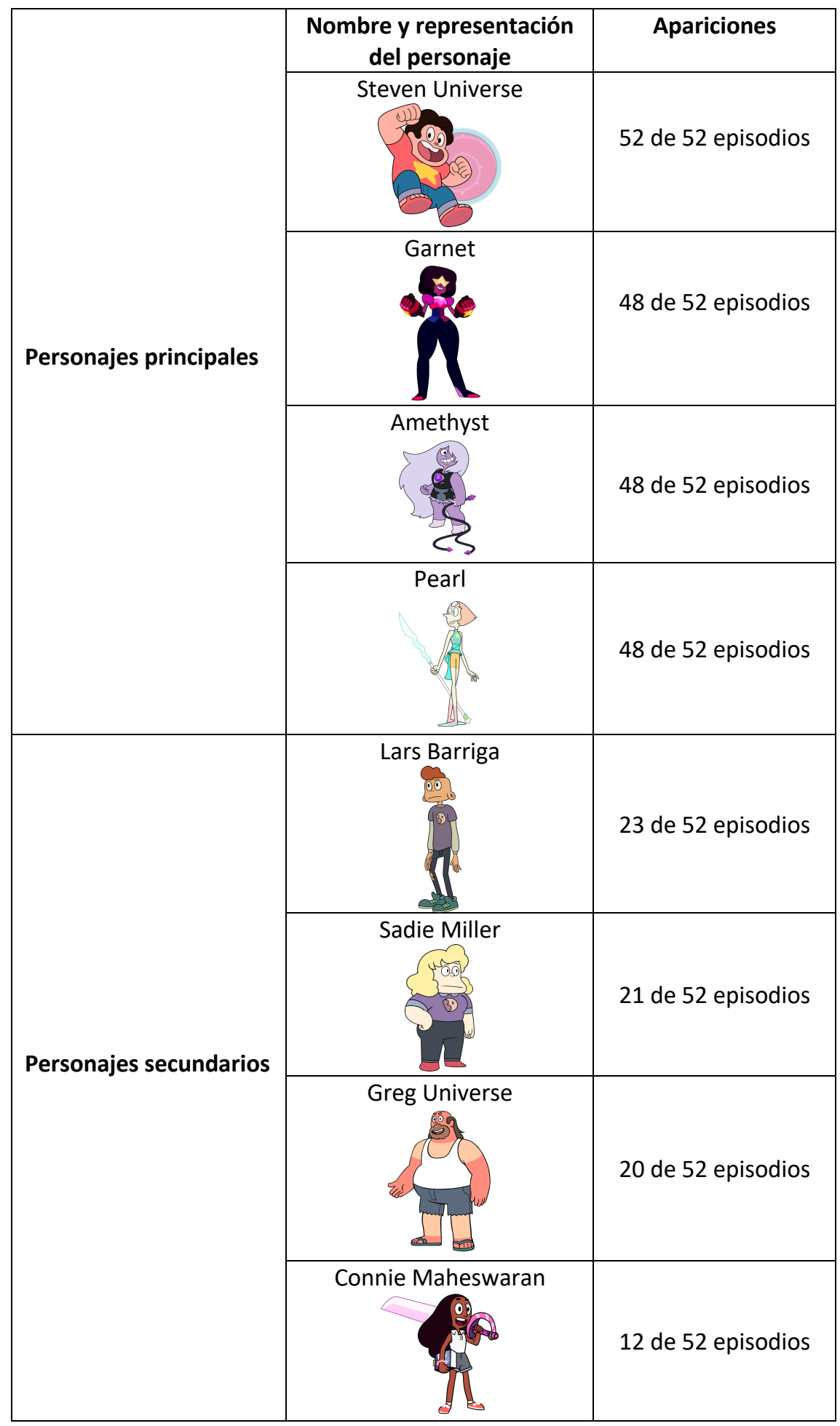

Fuente: Elaboración propia.

Las variables y categorías del libro de código necesario para el estudio han sido aportaciones de otros autores, entre los que destacamos las de Masanet, Medina y Ferrés (2012), Galán (2006), García-Muñoz y Fedele (2011) y GLAAD (2021). Las 
categorías principales son las siguientes: edad, aspecto físico, género, rasgos y tipos de personalidad, orientación sexual, actitud, temática, situación de convivencia y espacios representados (ver anexo 1).

\section{RESULTADOS}

La orientación sexual de los ocho personajes analizados se concreta en cinco heterosexuales y cisgénero ${ }^{7}$, entre los que se encuentra el protagonista principal Steven, y tres no heterosexuales y no binarios, que se corresponden a los demás personajes principales (cf. tabla 2$)$.

Tabla 2. Resultados según la orientación sexual, el género y la tipología del personaje

\begin{tabular}{|c|c|c|c|c|c|c|}
\hline \multirow{2}{*}{$\begin{array}{c}\text { Tipología de } \\
\text { personajes }\end{array}$} & \multicolumn{3}{|c|}{ Principales } & \multicolumn{3}{c|}{ Secundarios } \\
\cline { 2 - 6 } & cisgénero & $\begin{array}{c}\text { Mujer } \\
\text { cisgénero }\end{array}$ & $\begin{array}{c}\text { Persona no } \\
\text { binaria }\end{array}$ & $\begin{array}{c}\text { Hombre } \\
\text { cisgénero }\end{array}$ & $\begin{array}{c}\text { Mujer } \\
\text { cisgénero }\end{array}$ & $\begin{array}{c}\text { Persona } \\
\text { no } \\
\text { binaria }\end{array}$ \\
\hline Heterosexual & 1 & 0 & 0 & 2 & 2 & 0 \\
\hline No heterosexuales & 0 & 0 & 3 & 0 & 0 & 0 \\
\hline
\end{tabular}

Fuente: elaboración propia.

\subsection{CARACTERÍSTICAS FÍSICAS Y ORIENTACIÓN SEXUAL}

La mayor parte de los personajes de la ficción no se ajustan a los cánones de belleza de nuestra sociedad heteropatriarcal occidental. De hecho, más de la mitad de los elementos de la muestra seleccionada cumplen con la etiqueta "poco atractivos" y cuentan con un físico poco o nada normativo.

El 80\% de los personajes heterosexuales corresponde a la categoría "poco atractivo" y el único personaje que rompe esta tendencia es Connie, que es clasificada como "normal". En el caso de los personajes del colectivo LGTBIQ+, en cambio, la variedad de aspectos físicos es firmemente notoria. Pearl es el único personaje que entra en la categoría "atractivo / muy atractivo". Garnet en ciertos aspectos puede resultar muy hegemónico, pero en otros, todo lo contrario. Finalmente, Amethyst es el más alejado de los cánones de belleza.

\footnotetext{
7 Cisgénero: "Según Schilt y Westbrook (2009): Cis es el prefijo en latín que significa "en el mismo lado". Complementa a trans, el prefijo "a través de". "Cisgénero" sustituye los términos "no-transgénero" o "varón biológico" / "mujer biológica” para referirse a las personas cuyo sexo biológico, su cuerpo y su identidad personal coinciden." (Córdova, 2020, p. 112).
} 
Gráfico 1. Aspecto físico de los personajes heterosexuales

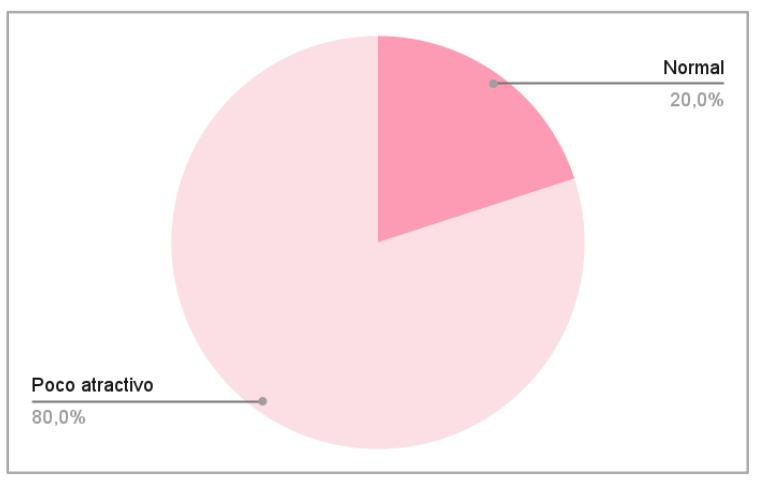

Fuente: elaboración propia.
Gráfico 2. Aspecto físico de los personajes del colectivo LGTBIQ+

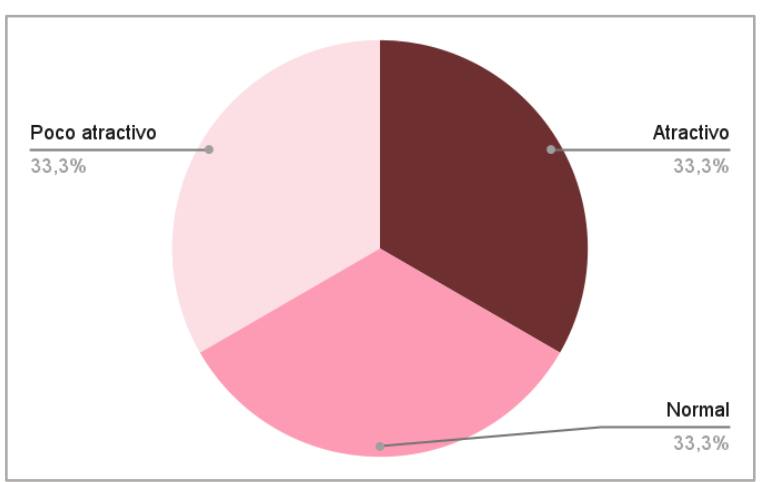

Fuente: elaboración propia.

\subsection{CARACTERÍSTICAS Y RASGOS DE LA PERSONALIDAD Y ORIENTACIÓN SEXUAL}

En líneas generales los rasgos de personalidad de los personajes para ambas categorías tienden a ser similares con algunas divergencias significativas.

Gráfico 3. Rasgos de personalidad de los personajes según su orientación sexual

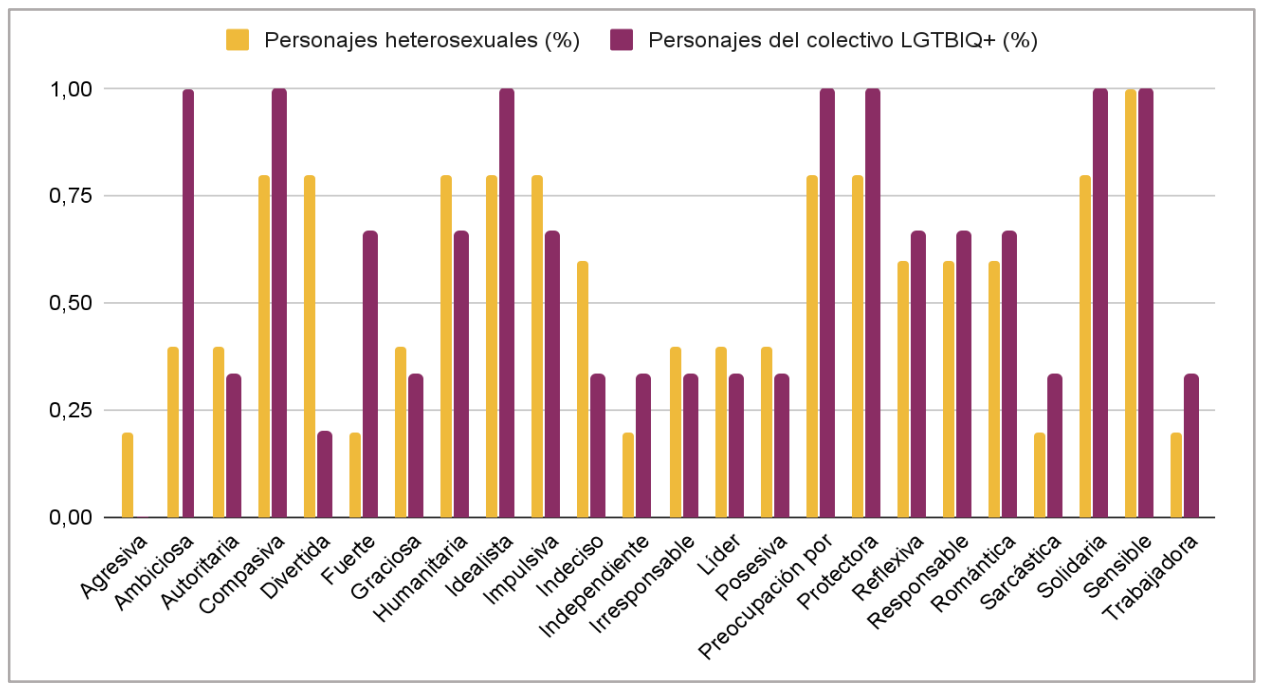

Fuente: elaboración propia.

En la muestra seleccionada a menudo la figura de Lars es la única que se diferencia de los otros personajes, ya que suele regirse por valores diferentes y adopta actitudes antagónicas a las del resto del elenco. ${ }^{8}$ La única diferencia realmente significativa se encontraría en la categoría "ambiciosa", ya que tan solo el $40 \%$ de los personajes

\footnotetext{
${ }^{8}$ De hecho si exceptuamos la categoría de "impulsiva", en los casos en que los valores de la columna de personajes heterosexuales es $80 \%$, esto se debe a que él no cumple con la característica.
}

RAE-IC, Revista de la Asociación Española de Investigación de la Comunicación vol. 8, núm. 16 (2021), 142-167 
heterosexuales obtendría un resultado positivo frente al $100 \%$ de los pertenecientes al colectivo LGTBIQ+.

En cambio, en cuanto a las actitudes que suelen adoptar los personajes, sí que se pueden observar ciertas diferencias.

Gráfico 4. Distribución de las actitudes de los personajes según la orientación sexual

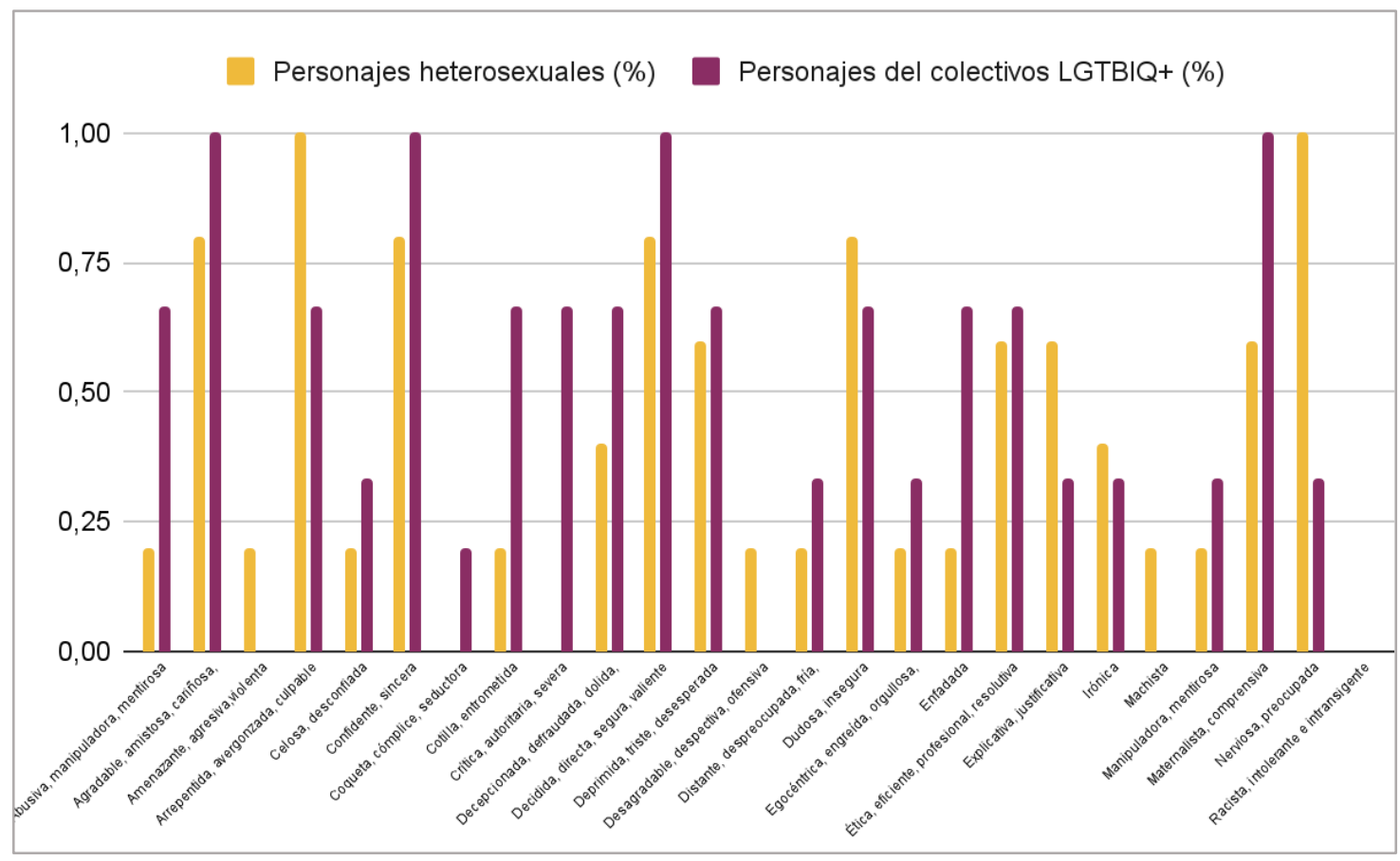

Fuente: elaboración propia.

La categoría de "nerviosa, preocupada" es una de las que presenta mayores disparidades entre un grupo y el otro, ya que se encuentra mucho más presente entre los personajes heterosexuales que entre los demás. En muchas ocasiones se representa a los personajes miembros del colectivo LGTBIQ+ como seres espiritualmente superiores, cuya edad es de varios miles de años.

La experiencia de estos personajes ayuda en parte a entender por qué la actitud "maternalista, comprensiva" es tan alta para este grupo, en comparación con el de los personajes heterosexuales. Pero también es importante tener en cuenta que estos tres personajes son los que conviven con Steven, lo educan y entrenan.

Los personajes del colectivo LGTBIQ+ muestran diferencias sustantivas en relación con categorías como "cotilla, entrometida", "crítica, autoritaria, severa" o "decepcionada, 
defraudada, dolida, frustrada". Esta fuerte desigualdad probablemente sea atribuible a que la presencia en pantalla de estos personajes también es considerablemente mayor que la de los demás (exceptuando el personaje homónimo de la ficción estudiada) y por eso también sus perfiles son mucho más completos y complejos.

En la muestra escogida el personaje de Garnet es sin duda el más fuerte de todos, y en varios pasajes el aspecto emocional es probablemente el más alejado de una caracterización humana.

Por otra parte, aquí aparece de forma clara que valores tales como la valentía ("decidida, valiente, segura, valiente"), la bondad ("agradable, amistosa, cariñosa, comprensiva" y "maternalista, comprensiva"), la confianza ("confidente, sincera") y la introspección o la reflexión ("arrepentida, avergonzada, culpable") son claves para el entendimiento de cómo se idearon y construyeron las representaciones de los personajes de Steven Universe.

El análisis de la muestra revela una actitud "desagradable, despectiva, ofensiva" y una "machista" en el personaje de Lars, ya en ciertas ocasiones tiende a cometer algunos micromachismos". No existen personajes con actitudes "racistas, intolerantes e intransigentes".

\subsection{EL CONTEXTO REPRESENTADO: TEMÁTICAS, ESCENARIOS Y ORIENTACIÓN SEXUAL}

La presencia de escenarios mágicos y no mágicos es bastante similar. Los escenarios "mágicos" no suelen ser visitados por más del 50\% de la muestra (los únicos en superarlo son la "Torre de Lapislázuli", la "Isla Aventura" y el "Teletransportador") y, exceptuando la "Playa", no hay ningún espacio en el que todos los personajes hayan estado incluidos o representados.

\footnotetext{
9 Micromachismo: "Los micromachismos son actitudes de dominación "suave" o de "bajísima intensidad", formas y modos larvados y negados de abuso e imposición en la vida cotidiana. Son, específicamente hábiles artes de dominio, comportamientos sutiles o indecisos, reiterativos y casi invisibles que los varones ejecutan permanente." (Bonino, 2004, p. 1).
} 
Gráfico 5. Porcentajes de elementos de la muestra que aparecen en cada escenario

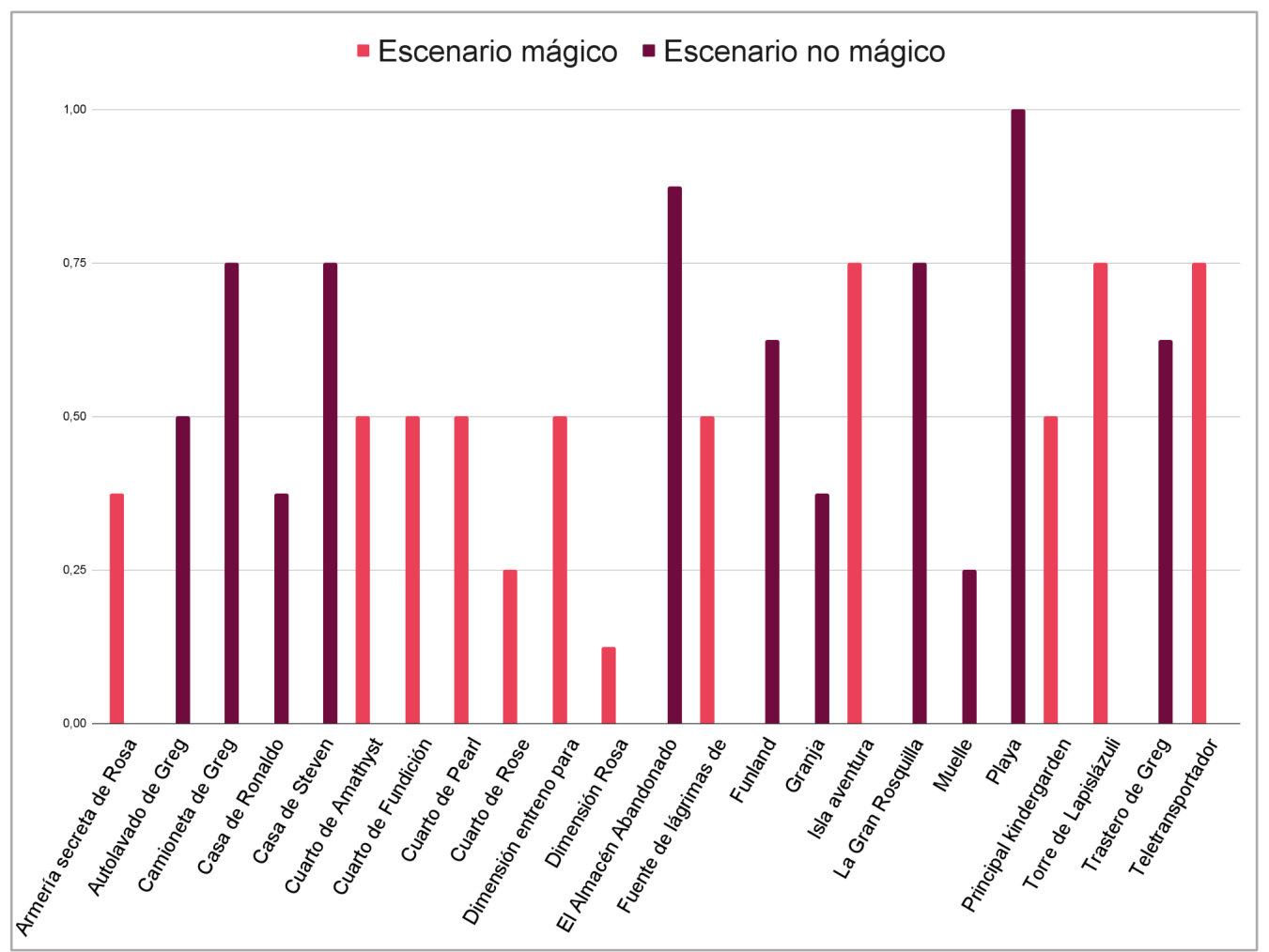

Fuente: elaboración propia.

Gráfico 6. Porcentajes de presencia de personajes en los diferentes escenarios según su orientación sexual

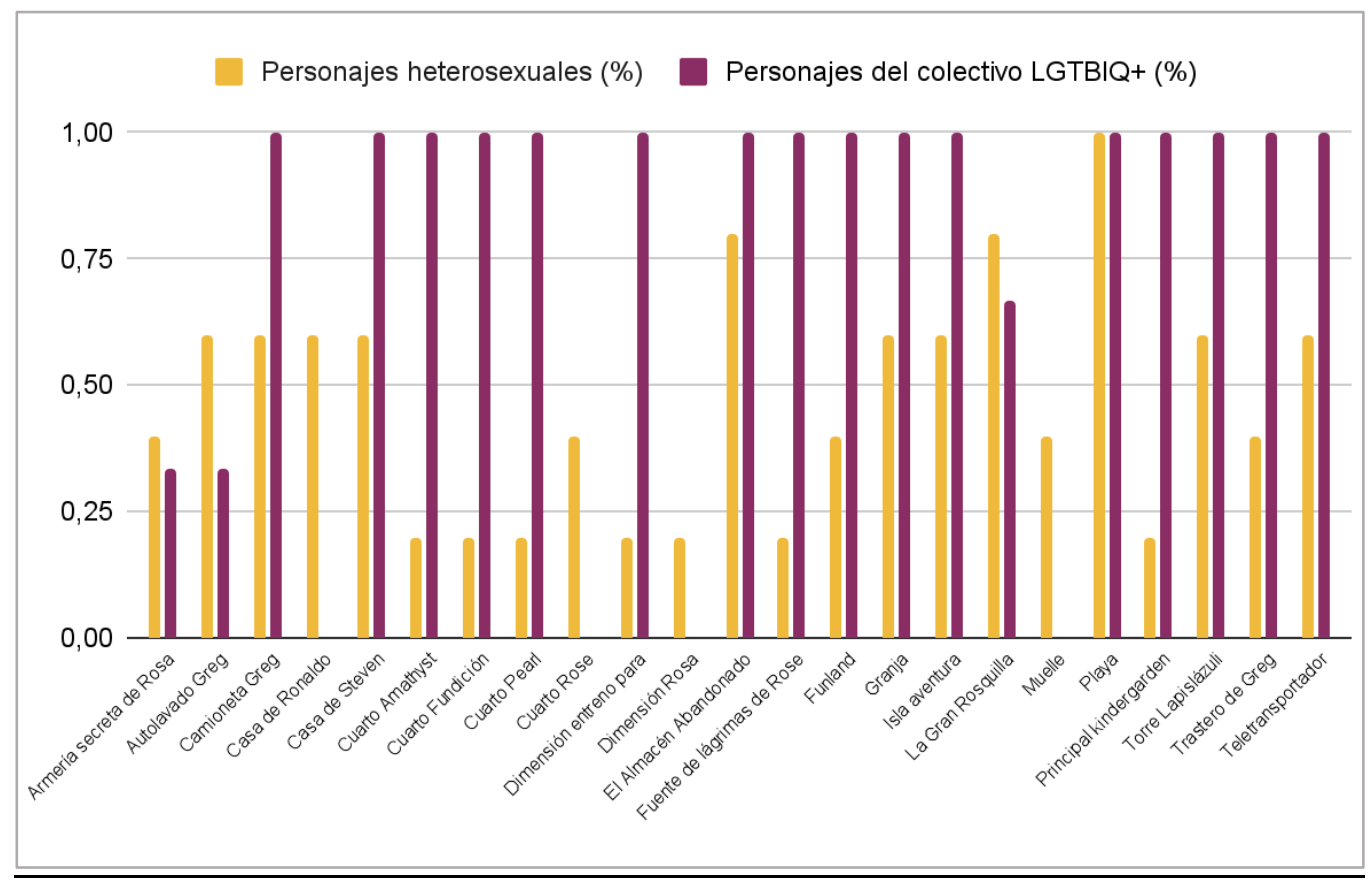

Fuente: elaboración propia.

RAE-IC, Revista de la Asociación Española de Investigación de la Comunicación vol. 8, núm. 16 (2021), 142-167 
Los personajes del colectivo LGTBIQ+ están más presentes que los demás en esta ficción seriada. La realidad es que, como ya hemos mencionado anteriormente, si omitimos el personaje de Steven, todos los demás personajes principales son del colectivo LGTBIQ+. Por otra parte, los cuatro personajes de la muestra que conforman la categoría de principales también son los cuatro únicos personajes-gemas y por lo tanto tienen poderes mágicos. Eso explicaría que en los escenarios mágicos su presencia sea casi siempre mayor que la de personajes heterosexuales.

En la mayoría de los casos los poderes mágicos representados en esta ficción animada están asociados a valores positivos, y los personajes que los poseen tienen como misión salvar el mundo.

\subsection{RASGOS PRINCIPALES DE LOS PERSONAJES LGTBIQ+.}

Todos los personajes pertenecientes al colectivo LGTBIQ+ tienen una personalidad "ambiciosa", "compasiva", "humanitaria", "idealista", "protectora” y que se "preocupa por los demás", "solidaria" y "sensible". Dos tercios de la muestra cuentan con una personalidad "fuerte", "reflexiva", "romántica" y "responsable".

Gráfico 7. Rasgos de personalidad presentes en los personajes del colectivo LGTBIQ+

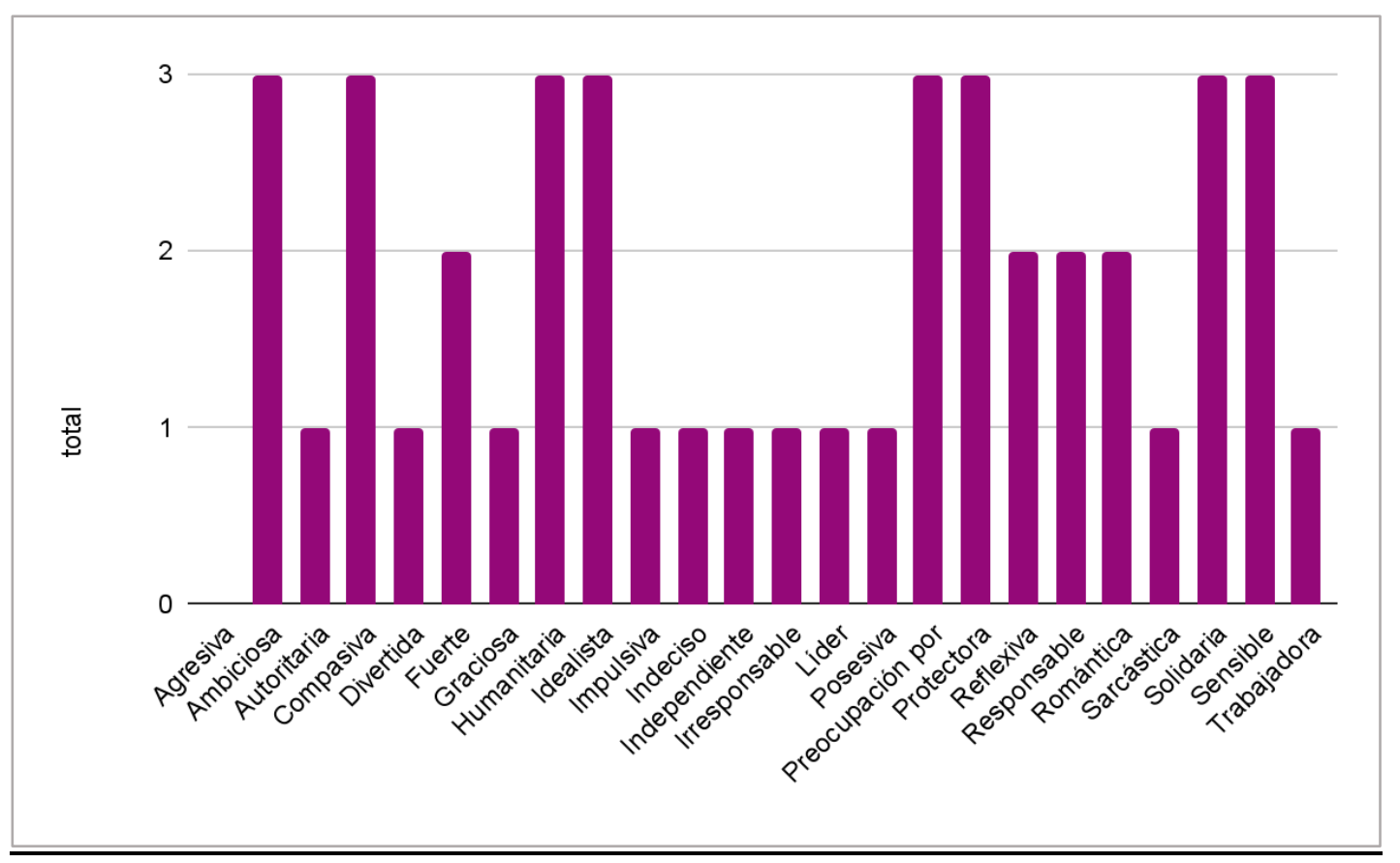

Fuente: elaboración propia.

RAE-IC, Revista de la Asociación Española de Investigación de la Comunicación vol. 8, núm. 16 (2021), 142-167 
Las actitudes que registran una mayor representación son "agradable, amistosa, cariñosa, comprensiva", "confidente, sincera", "decidida, directa, segura, valiente" y "maternalista, comprensiva". Mientras que los comportamientos menos reforzados son "celosa, desconfiada", "distante, despreocupada, fría" o "egocéntrica, engreída, orgullosa", entre otros.

Gráfico 8. Actitudes que suelen adoptar los personajes del colectivo LGTBIQ+

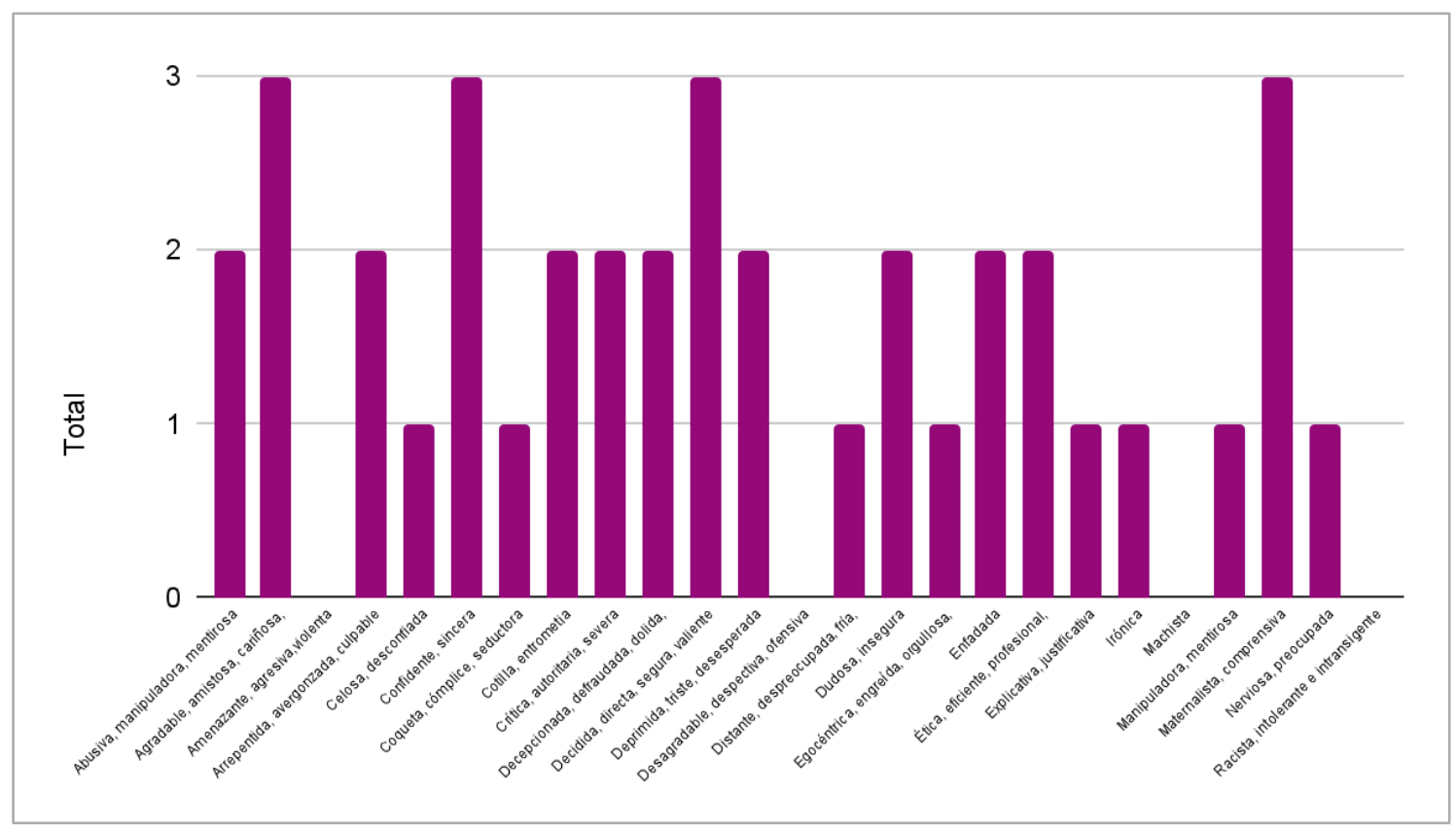

Fuente: elaboración propia.

Como podemos apreciar, todos los valores completamente ausentes en la muestra se caracterizan por ser atributos negativos de los personajes. En contraposición, las actitudes comunes a todos los personajes del colectivo LGTBIQ+ se corresponden con atributos positivos.

\section{CONCLUSIONES}

Los resultados del estudio nos permiten afirmar que los roles dominantes del retrato de los personajes LGBTIQ+ en Steven Universe se caracterizan por una marcada sensibilidad y sabiduría. Si bien no todos cuadran con la figura del líder, sí podemos indicar que todos se caracterizan por su valentía, su altruismo, filantropía y por luchar por sus ideales. Son representados como héroes, con superpoderes, pero sin perder sus rasgos, actitudes y 
sentimientos más humanos como podrían ser la inseguridad, la culpa, la tristeza, los celos, el amor, la amistad y expectativas de crecimiento personal.

Los sujetos heterosexuales tienden a ser más terrenales, con un aspecto físico bastante alejado de la heteronorma. Al tratarse de personajes jóvenes también podemos identificar rasgos y actitudes más propias de figuras con edades tempranas y aún en proceso de formación tanto personal como profesional o educacional.

Estos personajes nos permiten apreciar una clara evolución en la representación del colectivo LGTBIQ+ en los contenidos infantiles de la misma productora. A finales de la década de 1990 presentaban la figura de Him en las Powerpuff Girls que como bien explicaron Santos, Peres y Maranho (2019) fue una de las primeras representaciones queer que pudimos ver en contenidos infantiles. Progresivamente estos personajes fueron dejando este rol de villano. Con The Amazing World of Gumball, se dio un paso más allá en lo que respecta la inclusividad (Chiu y Chang, 2018). Pero Steven Universe revolucionó totalmente la animación infantil occidental en ese sentido, contando con personajes del colectivo LGTBIQ+, no solo con forma humana, sino que poniéndolos en el centro de la trama y haciéndolos su elenco protagónico (Delamorclaz, 2018; Robles 2020).

Esta presencia de personajes pertenecientes al colectivo LGTBIQ+ en ficción seriada y particularmente en ficción seriada infantil cubre una necesidad que muchos autores ya hace años han puesto sobre la mesa (Alfeo, 2011 y Martí, 2011). Hasta ahora los niños y niñas pertenecientes al colectivo LGTBIQ + no tenían personajes de ficción con los que pudieran contar como modelos o con los que sentirse identificados.

Como se puede leer en el último informe GLAAD (2020-2021), en los últimos años los contenidos audiovisuales para los más jóvenes han conocido un aumento bastante significativo en cuanto a la inclusión de personajes y temáticas relativas a la comunidad LGTBIQ+.

Si bien cabe preguntarse si los medios audiovisuales se inspiran en los estereotipos presentes en la sociedad para sus obras o si, por el contrario, son ellos los que construyen y difunden dichos estereotipos, a menudo ambos sectores se alimentan 
recíprocamente. En el caso de Steven Universe está claro que existe una voluntad de familiarizar al público más joven con la tolerancia e inclusión propias de una sociedad multifacética que promueva la aceptación de la diferencia y la convivencia en armonía.

En este sentido queda mucho camino por recorrer y muchos escenarios por conquistar en el terreno para lograr que la sociedad en su conjunto evolucione en el sentido de la aceptación y tolerancia al prójimo, algo que esta serie intenta difundir.

\section{AGRADECIMIENTOS}

Agradecemos a la doctora Rosa Franquet, a la doctora Claudia Contente, a Nicolás Fuertes, y al doctor Xavier Ribes Guardia por la lectura atenta y consejos recibidos.

\section{REFERENCIAS BIBLIOGRÁFICAS}

Aguado-Peláez, D. (2019). 'Steven Universe': un héroe dialógico como engranaje del cambio. Index.comunicación, 9(3), 207-235.

Alfeo, J. (2011). Análisis narratológico y sociedad representada: los personajes LGBT en el cine. En F. García García y M. Rajas (Eds.), Narrativas audiovisuales: los discursos (1aㅗ ed., pp. 63-84). Madrid: Icono14 editorial.

Bonino, L. (2004). Los Micromachismos. Revista La Cibeles, 2 (Ayuntamiento de Madrid). Recuperado de https://www.mpdl.org/sites/default/files/micromachismos.pdf

Buscarino. J (Productora) (2013-2019). Steven Universe [serie de televisión]. Cartoon Network Studios; Warner Bros. Television Distribution; HBO Entertainment.

Castro Bugarín, J. y Domingo, A. (2019). Diccionario LGTB+: Guía de conceptos de un lenguaje inclusivo. Agencia EFE. Recuperado de https://cutt.ly/4ReaduH

Chambers, S. A. (2009). The Queer Politics of Television. Editorial I.B. Tauris, 2009.

CHAT. (2021). Términos LGBTQ. Recuperado de https://www.chataboutit.org/terminoslgbtq/ 
Chiu, Y. y Chang, Y. (2018). Examining the Visual Styles and Visual Techniques in Animation Stories - A Case Study of The Amazing World of Gumball, 2018 1st IEEE International Conference on Knowledge Innovation and Invention (ICKII), 2018, pp. 324326. Recuperado de https://ieeexplore.ieee.org/document/8569144

Collins, R. (2009). The micro-sociology of violence. The British Journal Of Sociology, 60(3), 566-576. https://doi.org/10.1111/j.1468-4446.2009.01256.x

Cooley, K. (2020). Drawing queerness forward: Fusion, futurity, and Steven Universe. En. J. R. Ziegler y L. Richards (Eds.), Representation in Steven Universe (pp. 45-67) https://doi.org/10.1007/978-3-030-31881-9_3

Córdova Quero, H. (2020). Hacia un glosario queer: algunas nociones acerca del género, la sexualidad y la teoría queer.; Análisis, 52(96), 95-121.

Crenshaw, K. W. (1991). Mapping the margins: intersectionality, identity politics, and violence against women of color.; Stanford Law Review 43(6), 1241-1299.

Cuesta, U. y Menéndez, T. (2006). La percepción del personaje y su construcción psicológica. En F. García García (Ed.) Narrativa audiovisual. Madrid: Ediciones del Laberinto

Delamorclaz Ruiz, C. (2018). LGBTI y feminismo en animación televisiva: una reinterpretación de Steven Universe y Sailor Moon. Con A De Animación, 8, 164-177. https://doi.org/10.4995/caa.2018.9655

Delamorclaz Ruiz, C. (2017). Evolución del rol de las mujeres en la animación televisiva. III CONGRESO INTERNACIONAL DE INVESTIGACIÓN EN ARTES VISUALES, 1-3.

Recuperado de http://dx.doi.org/10.4995/ANIAV.2017.5771

Domínguez-Benítez, M. (2020). Una introducción a la teoría queer de Paul B. Preciado. En M. Domínguez-Benítez (Ed.), Investigaciones Feministas (pp. 91-101). Las Palmas de Gran Canaria: Ediciones Complutense 
Dunn, E. (2016). Steven Universe, Fusion Magic, and the Queer Cartoon Carnivalesque. En D. W. Riggs, C. Colton, C. Due, C. Bartholomaeus, R. Humphrey, E. Dunn y A. Anderson (Eds.), Transgender and the Media (pp. 44-58). Cologne: Universität zu Köln. Recuperado de https://cdn.atria.nl/ezines/IAV_606906/IAV_606906_2016_56.pdf\#page=47

Fellner, A. (2017). Trans Television Culture. Queer Politics, Gender Fluidity, and Quality TV. Oceánide, 9, 2017.

Fernández Paradas, A. R. (2017). La representación de las identidades sexuales en la serie Sense8 de los clichés socioculturales a la normalización sexual”. En La desigualdad de género invisibilizada en la comunicación: (aportaciones al III Congreso Internacional de Comunicación y Género y al I Congreso Internacional de Micro machismo en la comunicación), pp. 6-9. Editorial Dykinson.

Galán Fajardo, E. (2006). Personajes, estereotipos y representaciones sociales. Una propuesta de estudio y análisis de la ficción televisiva. ECO-PÓS, 9(1), 58-81.

García-García, A. y Cornelio-Marí, E. M. (2019). Adaptación cultural de Steven Universe para Hispanoamérica: exportación de una agenda progresista de género en animación. Global Media Journal México, 16(30), 1-26. https://doi.org/10.29105/gmjmx16.30-1

García-Muñoz, N. y Fedele, M. (2010). Las series televisivas juveniles: tramas y conflictos en una "teen serie". Comunicar, Revista Científica De Educomunicación, 37(XIX), 133-140.

García-Muñoz, N. y Martínez-García, L. (2009). The positive representation of the image of women in the media. [La representación positiva de la imagen de las mujeres en los medios]. Comunicar, 32, 209-214. https://doi.org/10.3916/c32-2009-03-002

Gerbner, G. et al. (2002). Growing up with Television: Cultivation Processes. En J. Bryant y D. Zillmann (Eds.). Media Effects. Advances in Theory and Research (pp. 4368). London: Taylor \& Francis.

GLAAD (2021). Where We Are on TV Report 2020-2021. Recuperado de https://cutt.ly/zRed4v9 
Lacalle, C. y Gómez, B. (2016). The representation of workingwomen in Spanish television fiction. [La representación de las mujeres trabajadoras en la ficción televisiva española]. Comunicar, 47, 59-67. https://doi.org/10.3916/C47-2016-06

Martí López, E. (2011). Homosexualidad, infancia y animación: Del nacimiento de Pebbles Picapiedra a la adopción de Ling Bouvier. Con A De Animación, 0(1), 97-118. https://doi.org/10.4995/caa.2011.863

Martín-Sánchez, D., Hernández-Herrarte, M. y Fernández-Ramos, M. (2020). El lenguaje no verbal como elemento constructor de estereotipos femeninos en la comedia de situación Modern Family. Revista Mediterránea De Comunicación/Mediterranean Journal Of Communication, 11(II), 277-293. https://www.doi.org/10.14198/MEDCOM2020.11.2.10

Martínez García, P. y Aguado-Peláez, D. (2017). La reapropiación de los cuerpos de las mujeres en la ficción televisiva. Análisis de Orange is the New Black. Investigaciones Feministas, 401-413. Ediciones Complutense.

Masanet Jordà, M. J., Medina Bravo P. y Ferrés i Prats, J. (2012). Representación mediática de la sexualidad en la ficción seriada dirigida a los jóvenes: estudio de caso de "Los Protegidos" y "Física o Química". Comunicación, 10(1), 1537-1548.

Pérez Heredia, M. (2016). Translating Gender Stereotypes: An Overview on Global Telefiction. En Otras Modernidades, 02/2016. Universidad de Milán.

Peterson, G.W. y Peters, D.F. (1983). Adolescents' Construction of Social Reality: The impact of Television and Peers. Youth and Society, 15(1), 67-85.

Pitre, J. (2020). Queer transformation, contested authorship, and fluid fandom. En. J. R. Ziegler y L. Richards (Eds.), Representation in Steven Universe (pp. 19-44). Cham: Palgrave Macmillan. https://doi.org/10.1007/978-3-030-31881-9_2

Robles, K. (2020). Representaciones de los personajes femeninos en series animadas: Los Picapiedra y Steven Universe. Policromias, 5, 49-86. 
Sánchez Sáinz, M. (2019). Pedagogías queer. ¿Nos arriesgamos a hacer otra educación? Madrid: Catarata.

Santos, G., Peres Mancio, C. y Maranho, E. (2019). Queer representation incorporated at "Him", character of "The Powerpuff Girls". Marginalized Narratives, 11(1).

Recuperado de https://revistas.ucp.pt/index.php/jsta/article/view/7321

Signorielli, N. y Bacue, A. (1999). Recognition and respect: A Content Analysis of Primetime Television Characters across three Decades. Sex Roles, 40(7-8), 527-544.

Zani, A. M. (2019). Poesía cuir contemporánea. Reescritura y reconfiguración de las identidades LGTBIQ: la construcción de una subjetividad lésbica cuir en la poesía de Micaela Szyniak. Escritos jóvenes dossier: "género y feminismos". 8(12).

Ziegler, J. R. y Richards, L. (2020). Representation in Steven Universe. Palgrave MacMillan

\section{ANEXOS}

Tabla 1. Libro de códigos

\begin{tabular}{|l|l|}
\hline \multicolumn{2}{|l|}{ Nombre del personaje } \\
\hline Dimensión física del personaje \\
\hline \multirow{2}{*}{ Edad $^{\mathbf{1 0}}$} & Niños y adolescentes \\
\cline { 2 - 2 } & Adultos \\
\hline \multirow{4}{*}{ Aspecto físico ${ }^{\mathbf{1 1}}$} & Atractivo / muy atractivo \\
\cline { 2 - 2 } & Normal \\
\cline { 2 - 2 } & Poco atractivo \\
\hline \multirow{5}{*}{ Género } & Hombre cisgénero \\
\cline { 2 - 2 } & Mujer cisgénero \\
\cline { 2 - 2 } & Hombre transgénero \\
\cline { 2 - 2 } & Mujer transgénero \\
\cline { 2 - 2 } & No binario \\
\cline { 2 - 2 } & Sin Identificar (SI) \\
\hline Gema & Sí \\
\cline { 2 - 2 } & No \\
\hline Dimensión psicológica del personaje \\
\hline Tipo de personalidad & Extrovertida \\
\hline
\end{tabular}

\footnotetext{
${ }^{10}$ Galán (2006)

${ }^{11}$ Galán (2006)

12 Galán (2006)
}

RAE-IC, Revista de la Asociación Española de Investigación de la Comunicación vol. 8, núm. 16 (2021), 142-167 


\begin{tabular}{|c|c|}
\hline & Introvertida \\
\hline \multirow[t]{24}{*}{ Rasgos de personalidad ${ }^{13}$} & Agresiva \\
\hline & Ambiciosa \\
\hline & Autoritaria \\
\hline & Compasiva \\
\hline & Divertida \\
\hline & Fuerte \\
\hline & Graciosa \\
\hline & Humanitaria \\
\hline & Idealista \\
\hline & Impulsiva \\
\hline & Indecisa \\
\hline & Independiente \\
\hline & Irresponsable \\
\hline & Líder \\
\hline & Posesiva \\
\hline & Preocupación por los demás \\
\hline & Protectora \\
\hline & Reflexiva \\
\hline & Responsable \\
\hline & Romántica \\
\hline & Sarcástica \\
\hline & Solidaria \\
\hline & Sensible \\
\hline & Trabajadora \\
\hline \multirow{9}{*}{$\begin{array}{l}\text { Temáticas con las que el } \\
\text { personaje suele verse } \\
\text { involucrado }^{14}\end{array}$} & Amistad \\
\hline & Amor \\
\hline & Aceptación personal \\
\hline & Discriminación \\
\hline & Expectativas de crecimiento personal \\
\hline & Pareja \\
\hline & Problemas familiares \\
\hline & Violencia \\
\hline & Económico \\
\hline \multirow{9}{*}{$\begin{array}{l}\text { Actitudes que suele } \\
\text { adoptar el personaje }\end{array}$} & Abusiva, manipuladora, mentirosa \\
\hline & Agradable, amistosa, cariñosa, comprensiva \\
\hline & Amenazante, agresiva, violenta \\
\hline & Arrepentida, avergonzada, culpable \\
\hline & Celosa, desconfiada \\
\hline & Confidente, sincera \\
\hline & Coqueta, cómplice, seductora \\
\hline & Cotilla, entrometida \\
\hline & Crítica, autoritaria, severa \\
\hline
\end{tabular}

${ }^{13}$ García-Muñoz y Fedele (2011)

${ }^{14}$ García Muñoz y Fedele (2011) Masanet, Medina y Ferrés (2012)

${ }^{15}$ Galán (2006)

RAE-IC, Revista de la Asociación Española de Investigación de la Comunicación vol. 8, núm. 16 (2021), 142-167 


\begin{tabular}{|c|c|}
\hline \multicolumn{2}{|c|}{\begin{tabular}{|l|l|} 
& Decepcionada, defraudada, dolida, frustrada
\end{tabular}} \\
\hline & Decidida, directa, segura, valiente \\
\hline & Deprimida, triste, desesperada \\
\hline & Desagradable, despectiva, ofensiva \\
\hline & Distante, despreocupada, fría, indiferente \\
\hline & Dudosa, insegura \\
\hline & Egocéntrica, engreída, orgullosa, prepotente \\
\hline & Enfadada \\
\hline & Ética, eficiente, profesional, resolutiva \\
\hline & Explicativa, justificativa \\
\hline & Irónica \\
\hline & Machista \\
\hline & Manipuladora, mentirosa \\
\hline & Maternalista, comprensiva \\
\hline & Nerviosa, preocupada \\
\hline & Racista, intolerante e intransigente \\
\hline \multicolumn{2}{|c|}{ Dimensión social del personaje } \\
\hline \multirow{2}{*}{$\begin{array}{l}\text { Estabilidad de las } \\
\text { relaciones }^{16}\end{array}$} & Estables \\
\hline & Cambiantes \\
\hline \multirow[t]{3}{*}{ Situación de convivencia } & Solo \\
\hline & Pareja amorosa \\
\hline & Compañeros de convivencia \\
\hline \multirow[t]{5}{*}{ Orientación sexual $^{17}$} & Heterosexual \\
\hline & Homosexual \\
\hline & Lesbiana \\
\hline & Bisexual \\
\hline & Sin Identificar (SI) \\
\hline \multicolumn{2}{|l|}{ Marco espacio-temporal } \\
\hline \multirow{16}{*}{$\begin{array}{l}\text { Espacios en los que } \\
\text { aparecen los personajes }\end{array}$} & Armería Secreta de Rose \\
\hline & Autolavado de Greg \\
\hline & Camioneta de Greg \\
\hline & Casa de Ronaldo \\
\hline & Casa de Steven \\
\hline & Cuarto de Amethyst \\
\hline & Cuarto de Fundición \\
\hline & Cuarto de Pearl \\
\hline & Cuarto de Rose \\
\hline & Arena de Cristal (Dimensión para los entrenamientos de Steven) \\
\hline & Dimensión Rosa \\
\hline & El Almacén Abandonado \\
\hline & Fuente de lágrimas de Rose \\
\hline & Funland \\
\hline & Granja \\
\hline & Isla Aventura \\
\hline
\end{tabular}

${ }^{16}$ Galán (2006)

${ }^{17}$ GLAAD (2020-2021)

RAE-IC, Revista de la Asociación Española de Investigación de la Comunicación vol. 8, núm. 16 (2021), 142-167 


\begin{tabular}{|l|l|}
\hline \multirow{4}{*}{} & La Gran Rosquilla \\
\cline { 2 - 3 } & Muelle \\
\cline { 2 - 3 } & Playa \\
\cline { 2 - 3 } & Principal Kindergarten \\
\cline { 2 - 3 } & Torre de Lapislázuli \\
\cline { 2 - 3 } & Trastero de Greg \\
\cline { 2 - 3 } & Teletransportador \\
\hline
\end{tabular}

Fuente: elaboración propia.

Tabla 2. Orientación sexual y tipología de los personajes

\begin{tabular}{|c|c|}
\hline Personajes principales & Personajes secundarios \\
\hline Steven: Heterosexual & Lars: Heterosexual \\
\hline Garnet: Lesbiana & Sadie: Heterosexual \\
\hline Amethyst: Bisexual & Greg: Heterosexual \\
\hline Pearl: Lesbiana & Connie: Heterosexual \\
\hline
\end{tabular}

Fuente: elaboración propia. 